\title{
BMJ Open Intelligence in young adulthood and alcohol use disorders in a prospective cohort study of Danish men: the role of psychiatric disorders and parental psychiatric history
}

\author{
Emilie Just-Østergaard, ${ }^{1}$ Trine Flensborg-Madsen, ${ }^{1}$ Joachim Knop, ${ }^{1}$ \\ Holger Jelling Sørensen, ${ }^{2}$ Ulrik Becker, ${ }^{3}$ Erik Lykke Mortensen ${ }^{1}$
}

To cite: Just- Østergaard E, Flensborg-Madsen T, Knop J, et al. Intelligence in young adulthood and alcohol use disorders in a prospective cohort study of Danish men: the role of psychiatric disorders and parental psychiatric history. BMJ Open 2019;9:e028997. doi:10.1136/ bmjopen-2019-028997

- Prepublication history for this paper is available online. To view these files, please visit the journal online (http://dx.doi. org/10.1136/bmjopen-2019028997).

Received 10 January 2019 Revised 02 August 2019 Accepted 09 August 2019

Check for updates

(C) Author(s) (or their employer(s)) 2019. Re-use permitted under CC BY-NC. No commercial re-use. See rights and permissions. Published by BMJ.

${ }^{1}$ Department of Public Health, University of Copenhagen, Copenhagen, Denmark ${ }^{2}$ Mental Health Centre Copenhagen, Copenhagen University Hospital Gentofte, Gentofte, Denmark

${ }^{3}$ National Institute of Public Health, University of Southern Denmark, Copenhagen, Denmark

Correspondence to Emilie Just-Østergaard; emju@sund.ku.dk

\section{ABSTRACT}

Objectives The aims were to estimate the association between intelligence measured in young adulthood and risk of alcohol use disorders (AUD) in men and to investigate the potential modification of this association by psychiatric disorders, parental AUD and parental psychiatric disorders.

Design Prospective cohort study based on a linkage of intelligence test scores from draft board examinations and register data on AUD diagnoses during 36 years of followup.

Setting Denmark.

Participants 3287 Danish men from the Copenhagen Perinatal Cohort (born 1959-1961) who appeared before the draft board at a mean age of 18.7 years.

Primary outcome measure First registration with AUD during follow-up was the primary outcome. Information on AUD was based on diagnoses retrieved from national hospital and outpatient treatment registers, defined according to the International Classification of Diseases. Results 361 (11.0\%) men were registered with AUD during follow-up. Low intelligence scores were associated with increased odds of AUD adjusting for parental AUD, parental psychiatric disorders, maternal smoking during pregnancy, birth weight, maternal age at birth, parity and childhood socioeconomic position (OR per SD decrease in intelligence $=1.69,95 \% \mathrm{Cl} 1.49$ to 1.92 ). Separate analyses indicated significant interaction $(p<0.001)$ between intelligence and psychiatric disorders. The adjusted $\mathrm{OR}$ per SD decrease in intelligence score was $2.04(95 \% \mathrm{Cl}$ 1.67 to 2.49 ) in men without other psychiatric disorders whereas the $0 \mathrm{R}$ was $1.21(95 \% \mathrm{Cl} 1.01$ to 1.46$)$ in men with other psychiatric disorders. No interaction was found between intelligence and parental AUD or between intelligence and parental psychiatric disorders.

Conclusions The association between intelligence in young adulthood and AUD is modified by other psychiatric disorders as low intelligence is primarily a risk factor for men without other psychiatric disorders. Future studies should take other psychiatric disorders into account when investigating associations between intelligence and AUD.

\section{Strengths and limitations of this study}

A sample of 3287 men from a Danish birth cohort with intelligence test scores from draft board examinations was followed for an average of 36 years.

- The prospective design and the possibility to combine information from different time points on both the included men and their parents are strengths of our study.

- Information on alcohol use disorders was based on national hospital and treatment registers; thus, men with unregistered alcohol use disorders were not included.

- Our study was based on men and it is unknown to what extent our findings apply to women.

\section{INTRODUCTION}

Findings from prospective studies suggest that high intelligence in childhood and young adulthood is protective against a range of somatic ${ }^{12}$ and psychiatric disorders. ${ }^{3-5}$ Likewise, growing evidence suggests that intelligence has a role in the aetiology of alcohol use disorders (AUD), but the findings have been conflicting. While some studies have shown a positive association between intelligence and alcohol problems, ${ }^{6}{ }^{7}$ other studies have not found an association. ${ }^{89}$ In the recent years, an inverse association has been reported between intelligence and alcohol-related outcomes. ${ }^{10-12}$ For example, an American study of male veterans showed that low intelligence was associated with a higher risk of AUD and other forms of psychopathology in midlife. ${ }^{11}$ Further, Swedish cohort studies have supported a robust inverse association between young adult intelligence and risk of alcohol-related morbidity and mortality. ${ }^{13-16}$ These conflicting findings may be due to differences in the measurement of 


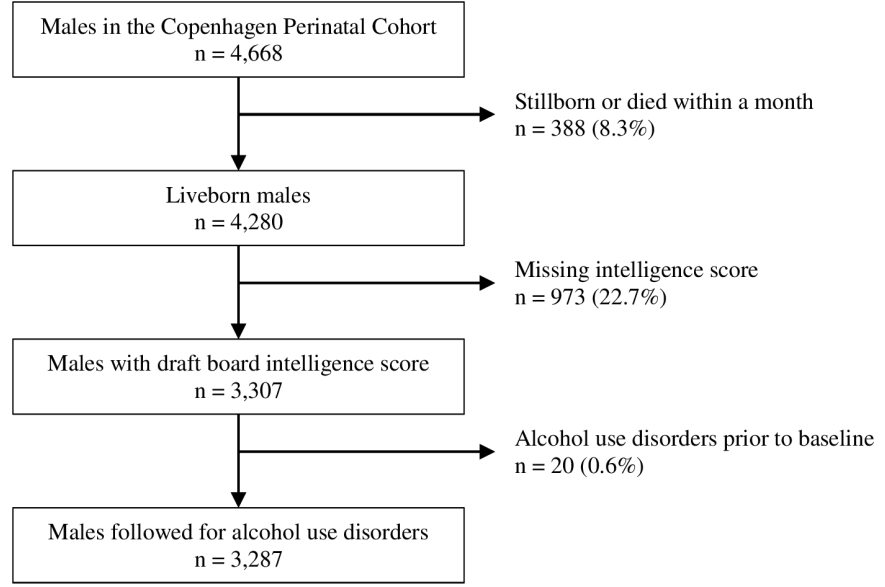

Figure 1 Flow chart of study population.

intelligence and AUD, but they also suggest that uninvestigated factors may influence and modify the intelligence-AUD association. Since other psychiatric disorders and parental psychiatric history are often associated with AUD,${ }^{17-20}$ they may be factors modifying the association of intelligence with AUD.

Few studies have considered the role of psychiatric disorders and parental psychiatric history in the association between intelligence in young adulthood and risk of AUD later in life. In Danish and Swedish conscript studies, the association between intelligence and alcohol-related disorders appeared robust to adjustment for psychiatric disorders ${ }^{5} 14$ and parental psychiatric history $^{515}$ and a large conscript study of Swedish men suggested little attenuating effect of excluding men with comorbid psychiatric disorders. ${ }^{16}$ However, none of the studies investigated the potential interaction with psychiatric disorders or parental psychiatric history making it impossible to address whether the effect of intelligence differs according to presence of such disorders. Further, there is little data on parental psychiatric history in this area and most studies have only had access to indicators of parental AUD such as parental drinking. ${ }^{14}$ An additional problem is that the potential confounding effect of early life factors such as parity, birth weight and health-related lifestyle during pregnancy in the intelligence-AUD association is relatively unexplored.

Mortensen et al conducted a prospective study on the Copenhagen Perinatal Cohort in 2005 and found a difference of -10.1 points (95\% CI -13.8 to -6.3 ) in average intelligence score for those with AUD compared with those without. ${ }^{5}$ Intelligence was measured with a validated draft board intelligence test, Børge Priens Prøve (BPP), at a mean age of about 19 years and 103 men were registered with AUD during 20 years of follow-up. In the current study, we use the same intelligence test and recently updated data on AUD and other psychiatric disorders. We aim to expand previous findings by estimating the risk of AUD according to young adult intelligence score, and by testing the potential modification of this association by psychiatric disorders, parental AUD and parental psychiatric disorders. Different mechanisms may lead to modification of the association between intelligence and AUD: We expect that psychiatric disorders are a complicating factor possibly reducing an association between intelligence and AUD. In contrast, a stronger association between intelligence and AUD is anticipated among individuals with parental AUD/parental psychiatric disorders as the protective effect of high intelligence may be particularly important in individuals with a parental psychiatric history.

\section{METHODS \\ Study population}

The analyses are based on data from the Copenhagen Perinatal Cohort and draft board intelligence test scores. The Copenhagen Perinatal Cohort is a Danish birth cohort comprising 8949 women and their 9125 children (51.2\% males; $48.8 \%$ females) born during 1959-1961 at the Copenhagen University Hospital. Most of the women were residents in Copenhagen, but some were admitted due to obstetrical complications or single mother status. ${ }^{21}$ Comprehensive information on early life factors was recorded prospectively during the pregnancy, at delivery and at a 1-year follow-up examination. ${ }^{22} \mathrm{~A}$ total of 8400 children (51.0\% males; $49.0 \%$ females) survived the first month after birth. Draft board intelligence test score was available for 3307 men (77.3\%). Men with a diagnosis of AUD $(n=20)$ prior to appearing before the draft board were excluded, resulting in a study population of 3287 men. The selection of the study population is illustrated in figure 1 .

\section{Patient and public involvement}

No participants were involved in the development of the research aim, data interpretation or the overall design of the study.

The current study is covered by permissions to the authors from the Danish Data Protection Agency.

\section{Draft board intelligence test}

All Danish men are required to appear before the draft board at the age of 18 years for assessment of their suitability for military service. Exempted from appearing are men with disqualifying diseases (eg, asthma and diabetes) and men who volunteered for military service before the age of 18. The draft board examination consists of a medical assessment and administration of an intelligence test, the BPP. ${ }^{23}$ It is a group-administered test consisting of four subtests: letter matrices (19 items), verbal analogies (24 items), number series (17 items) and geometric figures (18 items). The total BPP score (ranging from 0 to 78 ) is considered to be a high-quality measure of intelligence and it correlates 0.82 with the full-scale Wechsler's Adult Intelligence Scale. ${ }^{24}$ Based on the full study sample, the BPP total scores were standardised to a scale with a mean of 100 and an SD of 15 to correspond with the most widely used intelligence scales. 


\section{Alcohol use disorders}

To obtain information on AUD, data on the men were linked to three national registers by means of the unique personal identification number. ${ }^{25}$ The Danish Psychiatric Central Register contains data on all admissions to Danish psychiatric inpatient facilities since $1969 .^{26}$ The Danish National Patient Register contains records of all Danish hospital admissions since $1977 .^{27}$ Both registers have included diagnoses of outpatients since 1995. In the two registers, diagnoses were classified according to WHO's International Classification of Diseases (ICD; 8th revision until 1994, and 10th revision since 1994). The men were followed in the two registers until 21 June 2015. The Copenhagen Alcohol Cohort contains data on all individuals treated for AUD in outpatient facilities in greater Copenhagen since $1954 .{ }^{28}$ The men were followed in this register until 6 April 2009, when registration stopped.

We defined AUD as first hospital admission with a relevant diagnosis: ICD-8 (303: alcoholism), ICD-10 (F10.1: harmful use, F10.2: dependence syndrome) or by firsttime registration in the Copenhagen Alcohol Cohort, whichever occurred first.

\section{Psychiatric variables}

We extracted information from various national registers to define three psychiatric variables: Psychiatric disorders (yes and no) in the men were obtained from the Danish Psychiatric Central Register and the Danish National Patient Register and defined as first admission with a psychiatric disorder other than AUD (ICD-8: 292-302, 304-315; ICD-10: F11-F99). Parental psychiatric history comprised parental AUD and parental psychiatric disorders. Information on parental AUD was obtained from the Danish Psychiatric Central Register, The Danish National Patient Register and the Copenhagen Alcohol Cohort. Parental AUD was defined as a binary variable (yes and no) with yes representing having one or both parents registered with one of the following diagnoses: ICD-8 (303: alcoholism), ICD-10 (F10.1: harmful use, F10.2: dependence syndrome) or by first-time registration in the Copenhagen Alcohol Cohort. Parental psychiatric disorders were obtained from the Danish Psychiatric Central Register and defined as a binary variable (yes and no) with yes representing having one or both parents registered with a psychiatric disorder other than AUD (ICD-8: 292-302, 304-315; ICD-10: F11-F99).

\section{Confounders}

We selected the following early life covariates as potential confounders of the intelligence-AUD association since they have been associated with intelligence and alcohol-related outcomes: Maternal smoking in the final trimester of pregnancy (yes and no), birth weight (grams), maternal age at birth (years), parity and childhood socioeconomic position (SEP). Childhood SEP was measured as a composite indicator (1-8 point scale, low to high) based on the breadwinner's occupation, breadwinner's education, type of income (weekly or monthly) and quality of housing. All potential confounders were measured at birth or the 1-year examination of the Copenhagen Perinatal Cohort.

\section{Statistical analyses}

Initial analyses calculated descriptive statistics of the covariates according to intelligence score categories $(<90$, 90-110 and $>110$ points). Logistic regression models were used to estimate the association between intelligence and risk of AUD. We decided to analyse data by logistic regression models instead of survival analysis because the time of AUD registration may differ substantially from the actual time of development of AUD. In these analyses, intelligence was modelled as a continuous measure and ORs were computed for every $1 \mathrm{SD}$ decrease $(\mathrm{SD}=15)$ in intelligence score. The assumption of linearity was evaluated by fitting loess regression models, but no violations were observed. We used three statistical models on the full sample: (1) unadjusted, (2) adjusted for parental AUD and parental psychiatric disorders and (3) adjusted for parental AUD, parental psychiatric disorders, maternal smoking during pregnancy, birth weight, maternal age at birth, parity and childhood SEP.

To evaluate the potential modification of the effect of intelligence by psychiatric disorders in the men as well as by parental AUD and parental psychiatric disorders, we tested the interaction between each of the psychiatric variables and intelligence. The interaction was significant between psychiatric disorders and intelligence with respect to risk of AUD $(\mathrm{p}<0.001)$ and this was further examined in separate analyses stratified by psychiatric disorders. In contrast, no significant interaction was found between intelligence and parental AUD ( $p=0.28)$ or between intelligence and parental psychiatric disorders $(\mathrm{p}=0.35)$.

The rate of missing data on covariates ranged from $0 \%$ (psychiatric disorders and birth weight) to $19.4 \%$ (childhood SEP). The highest proportion of missing data concerned childhood SEP (19.4\%), parental AUD $(10.9 \%)$ and parental psychiatric disorders $(10.9 \%)$. The relatively high proportion of missing on childhood SEP is due to the fact that it was measured at the 1-year examination of the Copenhagen Perinatal Cohort with some drop-out, whereas the missing data on parental psychiatric history (parental AUD and parental psychiatric disorder) are due to lacking personal identification numbers on some parents. To deal with missing data on covariates, we conducted all analyses using multiple imputation where missing values are estimated based on the distribution of other variables in the dataset. ${ }^{29}$ Multiple imputation was performed using 40 imputed datasets. In sensitivity analyses, complete case analyses were also conducted using non-imputed data. It could be expected that the association between intelligence and AUD would differ by the age of onset of AUD (eg, early vs late registration of AUD). In sensitivity analyses, we analysed the association between intelligence and AUD among those registered 
Table 1 Descriptive statistics of the 3287 men according to intelligence test score: the Copenhagen Perinatal Cohort 19591961, with follow-up until 2015, Denmark

\begin{tabular}{|c|c|c|c|c|}
\hline & & elligence test & ore & \\
\hline & $\begin{array}{l}<90 \\
(n=800)\end{array}$ & $\begin{array}{l}90-110 \\
(n=1586)\end{array}$ & $\begin{array}{l}>110 \\
(n=901)\end{array}$ & P value* \\
\hline Psychiatric disorders, $\mathrm{n}(\%) \dagger$ & & & & \\
\hline Yes & $227(28.4)$ & $269(17.0)$ & $96(10.7)$ & $<0.001$ \\
\hline No & $573(71.6)$ & $1317(83.0)$ & $805(89.4)$ & \\
\hline Parental alcohol use disorders, $\mathrm{n}(\%)$ & & & & \\
\hline Yes & $108(15.7)$ & $151(10.6)$ & $46(5.6)$ & $<0.001$ \\
\hline No & $582(84.4)$ & $1270(89.4)$ & $771(94.4)$ & \\
\hline Parental psychiatric disorders, $\mathrm{n}(\%) \dagger$ & & & & \\
\hline Yes & $217(31.5)$ & $382(26.9)$ & $166(20.3)$ & $<0.001$ \\
\hline No & $473(68.6)$ & $1039(73.1)$ & $651(79.7)$ & \\
\hline Maternal smoking during pregnancy, n (\%) & & & & \\
\hline Yes & $462(59.5)$ & $795(51.5)$ & $371(41.9)$ & $<0.001$ \\
\hline No & $314(40.5)$ & $749(48.5)$ & $514(58.1)$ & \\
\hline Birth weight (grams), mean (SD) & $3179(660.7)$ & $3275(622.7)$ & $3373(605.1)$ & $<0.001$ \\
\hline Maternal age at birth (years), mean (SD) & $25.3(6.70)$ & $25.4(6.33)$ & $27.5(6.37)$ & $<0.001$ \\
\hline Parity, first pregnancy, $\mathrm{n}(\%)$ & & & & \\
\hline Yes & $366(45.8)$ & 835 (52.7) & $468(52.1)$ & 0.004 \\
\hline No & 433 (54.2) & $749(47.3)$ & $430(47.9)$ & \\
\hline Childhood socioeconomic position, mean (SD) $\ddagger$ & $3.1(1.44)$ & $3.9(1.69)$ & $5.1(1.86)$ & $<0.001$ \\
\hline
\end{tabular}

${ }^{*} \mathrm{P}$ value for $\chi^{2}$ tests or ANOVA.

†The variable included registration of psychiatric disorders other than alcohol use disorders.

łLinear component (1-8 score with higher points indicating higher childhood socioeconomic position).

ANOVA, analysis of variance.

before and after the median AUD age ( $<36$ vs $\geq 36$ years). All analyses were performed in SAS V.9.4.

\section{RESULTS}

\section{Descriptive results}

The study population comprised 3287 men, who appeared before the draft board at the mean age of 18.7 years. Characteristics of the men according to intelligence score are shown in table 1 . All the psychiatric variables and confounders were significantly associated with intelligence score. Thus, psychiatric disorders, parental AUD and parental psychiatric disorders were associated with low intelligence. Also, low intelligence score was associated with maternal smoking during pregnancy, lower birth weight, lower maternal age at birth, not being the first pregnancy and lower childhood SEP.

During 36 years of follow-up, 361 men (11.0\%) were registered with AUD. The median age of first AUD registration was 36.0 years. Among men with AUD, 34\% were registered between ages 18 and 30,32\% between ages 30 and 40 and $33 \%$ between ages 40 and 54 . The standardised intelligence score ranged from 53 to 144 points and the mean score was $91.3(\mathrm{SD}=14.52)$ among men with AUD and 101.1 ( $\mathrm{SD}=14.68)$ among men without AUD.
The unadjusted mean difference in intelligence score was -9.9 points $(95 \%$ CI -11.5 to -8.3$)$ between men with and without AUD corresponding to about two-thirds of an SD difference.

\section{Full sample}

Table 2 shows the ORs of being registered with AUD among men according to intelligence score, parental AUD and parental psychiatric disorders. Lower intelligence scores were associated with higher odds of AUD. In the unadjusted model, a $1 \mathrm{SD}$ decrease in intelligence score $(\mathrm{SD}=15)$ was associated with $93 \%$ higher odds of developing AUD (OR 1.93, 95\% CI 1.73 to 2.16). Adjustment for parental AUD and parental psychiatric disorders attenuated the OR slightly to 1.89 (95\% CI 1.68 to 2.11). When adjusting for all confounders in model 3 , a $1 \mathrm{SD}$ decrease in intelligence score was associated with $69 \%$ higher odds of developing AUD (OR 1.69, 95\% CI 1.49 to 1.92 ).

With respect to parental psychiatric history in the multiple adjusted model, no significant association was found between parental AUD and AUD in the men. In contrast, parental psychiatric disorders were associated with higher odds of AUD. 
Table 2 ORs of alcohol use disorders in relation to intelligence test score and parental psychiatric history, in the full sample of 3287 Danish men and in stratified analyses*

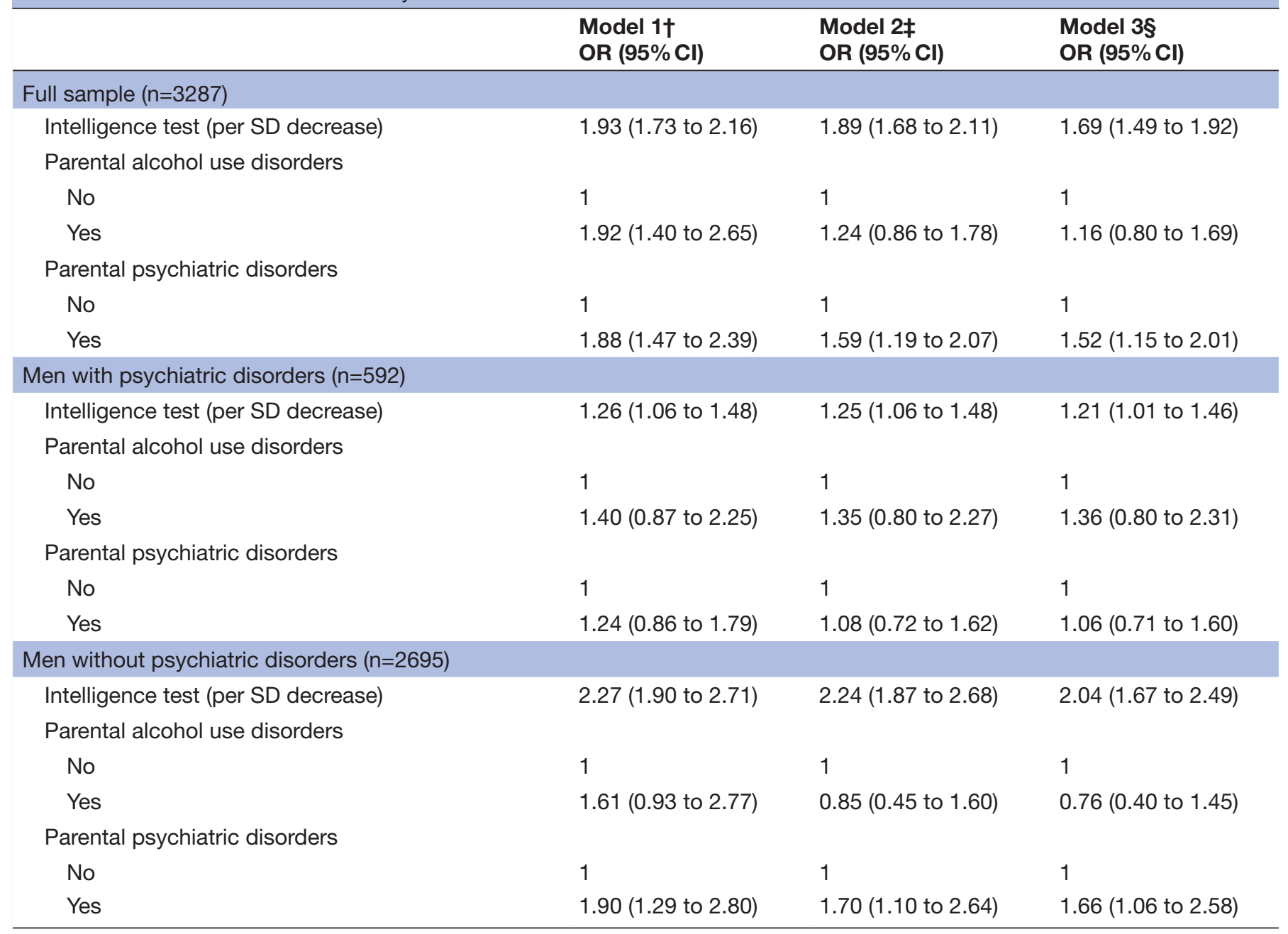

*The interaction between intelligence and other psychiatric disorders with respect to risk of alcohol use disorders was statistically significant ( $p$ value for interaction $<0.001$ ).

†Model 1 was unadjusted.

$\ddagger$ Model 2 included intelligence, parental alcohol use disorders and parental psychiatric disorders.

§Model 3 included intelligence, parental alcohol use disorders, parental psychiatric disorders, maternal smoking during pregnancy, birth weight, maternal age at birth, parity and childhood socioeconomic position.

\section{Stratified analyses}

Due to significant interaction between intelligence and other psychiatric disorders $(\mathrm{p}<0.001)$ with respect to risk of AUD in the multiple adjusted model, the full sample was stratified by psychiatric disorders (table 2). The group with other psychiatric disorders comprised 592 men and $222(38 \%)$ developed AUD, whereas the group without other psychiatric disorders consisted of 2695 men and 139 $(5 \%)$ developed AUD. In the stratified analyses, the association between intelligence and AUD remained significant in both groups comprising men with and without other psychiatric disorders, but low intelligence had the largest influence on risk of AUD in the group without psychiatric disorders. The multiple adjusted OR associated with $1 \mathrm{SD}$ decrease in intelligence score was 1.21 (95\% CI 1.01 to 1.46$)$ in men with psychiatric disorders, whereas the corresponding OR was 2.04 (95\% CI 1.67 to 2.49) in men without psychiatric disorders.

Regarding parental psychiatric history in the stratified analyses, we found significant association between parental psychiatric disorders and AUD in the group without other psychiatric disorders exclusively (OR 1.66, 95\% CI 1.06 to 2.58 ).

Sensitivity analyses with non-imputed data revealed no substantial changes in the results, neither in the full sample nor in the stratified analyses (data not shown). To determine whether the association between intelligence and AUD differed according to the age of onset, we divided cases of AUD into those registered before and after age 36 . The OR associated with 1 SD decrease in intelligence score was 2.57 (95\% CI 2.18 to 3.01) in men registered with AUD before age 36, whereas the 
corresponding OR was 1.47 (95\% CI 1.27 to 1.71) in men registered with AUD after age 36. Thus, low intelligence was associated with higher odds of AUD in both age groups, although stronger associations may exist among those registered with AUD early in life.

\section{DISCUSSION}

This cohort of 3287 men with 36 year follow-up showed that intelligence in young adulthood was associated with an increased risk of AUD later in life. The association was not explained by adjustment for parental psychiatric history and potential confounders measured early in life such as birth weight and childhood SEP. Separate analyses suggested that psychiatric disorders moderate the effect of intelligence on risk of AUD. Hence, intelligence was associated with a twofold higher risk of AUD in men without other psychiatric disorders, whereas a weaker association was found in men with other psychiatric disorders. Contrary to what was anticipated, parental AUD and parental psychiatric disorders did not appear to interact with intelligence.

\section{Comparisons and interpretations}

Our findings of a higher risk of AUD according to lower draft board intelligence test score in men are in line with prospective conscript studies suggesting an inverse association between intelligence and alcohol-related disorders. ${ }^{11}{ }^{13-15}$ A possible explanation for the observed protective effect of high intelligence is that more intelligent people are better able to handle a prolonged high intake of alcohol without actually developing AUD. Some prospective studies have shown that more intelligent people are less likely to abstain from alcohol, are more likely to have higher alcohol intake and to drink frequently in adult life. ${ }^{730}{ }^{31}$ It is likely that people with greater cognitive resources are better at assessing when their drinking behaviour is getting risky and they may also be better at assessing how to reduce their alcohol intake. Such an explanation is in keeping with literature suggesting that the ability to change one's own health behaviour is associated with cognitive ability. ${ }^{33}$ Alternatively, more educated and intelligent individuals may be more likely to hide their alcohol use problems from health professionals and less likely to ask for help, and therefore, not receive a diagnosis. It could also be that health professionals may tend to overlook AUD among more educated individuals and attribute the symptoms to less stigmatising conditions.

We were able to expand on previous findings by including a broad range of confounders measured early in life. Additional adjustment for parental AUD, parental psychiatric disorders, maternal smoking during pregnancy, birth weight, maternal age at birth, parity and childhood SEP led to modest reductions, suggesting that these early life factors have a limited impact on the intelligence-AUD association.
While few studies have adjusted for psychiatric disorders to estimate the influence of intelligence on risk of AUD, ${ }^{14}$ we suggest that other psychiatric disorders may act as an effect modifier and that this should be taken into account when analysing intelligence-AUD associations. Specifically, whereas intelligence is a risk factor for AUD in men without other psychiatric disorders, the association was considerably weaker in men with other psychiatric disorders. The finding that intelligence does not influence the risk of AUD substantially among those registered with psychiatric disorders is intriguing. It is possible that we do not have enough statistical power to estimate an association among those with psychiatric disorders, but this seems unlikely as AUD was more common in men with other psychiatric disorders than in men without. Conversely, men with psychiatric disorders may comprise a high-risk group for AUD as they have comorbid diagnoses such as mood disorders and personality disorders, which are strongly associated with AUD. ${ }^{19} 3435$ Hence, the influence of other risk factors such as intelligence seems to play a less important role relative to their psychiatric condition. The presence of a psychiatric disorder has been hypothesised to interact with intelligence in relation to risk of other health-related outcomes than AUD. For instance, Batty et al showed strong associations between low intelligence and increased risk of attempted suicide among men without psychosis, whereas no marked association existed among men with psychosis. ${ }^{36}$

We also considered the risk of AUD according to parental psychiatric history and found that parental psychiatric disorders were associated with higher risk of AUD, whereas no association was found between parental AUD and AUD in the fully adjusted model. The latter finding was surprising given that Sørensen $e t$ al showed strong associations of both maternal and paternal AUD with risk of AUD in male offspring in their study on the Copenhagen Perinatal Cohort. ${ }^{18}$ However, Sørensen et al did not adjust for intelligence and early life factors (eg, birth weight) in their analyses which may explain the apparent lack of association between paternal AUD and AUD in our analyses. Our findings indicate that the broader measure of parental psychiatric disorders may be a better predictor for the development of AUD than more specific alcohol-related parental diagnoses.

\section{Strengths and limitations}

Our analyses were based on 3287 men from the Copenhagen Perinatal Cohort followed for 36 years. The prospective design and the possibility to combine information from different time points on both participants and their parents are main strengths of our study. Thus, the data on intelligence were collected prospectively in addition to important covariate information such as other psychiatric disorders, parental AUD, parental psychiatric disorders and childhood SEP. Also, information on intelligence was collected relatively early in life (mean age 18.7 years) reducing the risk of early undiagnosed AUD to substantially influence intelligence scores. 
Our study also has limitations. As AUD diagnoses were based on registers, we were not able to include men with unregistered AUD. Thus, some persons may have fulfilled the criteria for an alcohol diagnosis without being in contact with a Danish hospital or an outpatient treatment facility during follow-up. Therefore, the men identified with AUD in our study are likely to be those with more severe forms of AUD and it can be debated whether our findings apply to persons not registered at a hospital. Further, we did not consider trajectories of AUD across the lifespan in relation to intelligence scores, although recent studies indicate that AUD varies across the lifespan. ${ }^{37} 38$ Nevertheless, our sensitivity analyses according to AUD onset indicated that intelligence may have a larger influence on early-onset AUD than lateonset AUD. Some evidence of positive assortative mating in relation to risk of AUD and other psychiatric disorders exist, ${ }^{39}$ but as the partners' AUD status was unknown in our study, we do not know how this factor would have affected our results. It could be that positive assortative mating represents an additional risk factor potentially obscuring the detrimental influence of low intelligence. Additionally, as our analyses are based on middle-aged Danish men (53-55 years at end of follow-up), our results do not necessarily apply to older men or to women. In this study, we had extensive information on early life factors, parental AUD and parental psychiatric disorders. Yet, residual confounding from parental AUD and parental psychiatric disorders cannot be fully excluded, as some parents are likely to have developed a psychiatric condition before the beginning of the registers in the 1970s and because not all psychiatric conditions will lead to registration. Also, we cannot reject confounding by unmeasured factors; for instance, early adverse experiences and genetic factors, which influence both development of intelligence ${ }^{40} 41$ and the risk of later AUD, ${ }^{42-44}$ were not included. Regarding other psychiatric disorders in the men, we were not able to distinguish between subcategories of psychiatric disorders because of too few cases in the subcategories. A larger sample size would have enabled us to study interactions of intelligence with separate psychiatric categories.

\section{CONCLUSION}

While AUD is considered to be a major public health challenge throughout the lifespan, the causes of AUD are not well documented. This cohort study supports a robust association between low intelligence measured in young adulthood and risk of AUD later in life and adds to the sparse literature by providing insight into the role of other psychiatric disorders. Thus, separate analyses suggest that associations between intelligence and AUD are primarily restricted to men without psychiatric disorders, whereas evidence of an intelligence-AUD association is weaker in men with psychiatric disorders. Given the novelty of these findings, studies should evaluate the role of other psychiatric disorders and parental psychiatric disorders when analysing associations of intelligence with AUD.

Acknowledgements We acknowledge the crucial role of $A L$ Willumsen and $B$ Zachau-Christiansen in the establishment of the Copenhagen Perinatal Cohort and thank the Steering Committee for permission to conduct the current study.

Contributors EJ- $\emptyset$, TF-M and ELM contributed to the conception and design of the study, performed the data analyses and prepared the first draft. JK, HJS and UB contributed to the interpretation of data and critically revised and reviewed the first draft. All authors read and approved the final manuscript.

Funding This work was supported by Innovation Fund Denmark, Health and Clinical Research (603-00520B).

Disclaimer The funder had no role in the study design, data collection and analysis, decision to publish, or preparation of the manuscript.

Competing interests None declared.

Patient consent for publication Not required.

Ethics approval Because our study was register based and did not require any contact with participants, no ethics approval was required according to Danish legislation.

Provenance and peer review Not commissioned; externally peer reviewed. Data availability statement № data are available.

Open access This is an open access article distributed in accordance with the Creative Commons Attribution Non Commercial (CC BY-NC 4.0) license, which permits others to distribute, remix, adapt, build upon this work non-commercially, and license their derivative works on different terms, provided the original work is properly cited, appropriate credit is given, any changes made indicated, and the use is non-commercial. See: http://creativecommons.org/licenses/by-nc/4.0/.

\section{REFERENCES}

1. Batty GD, Mortensen EL, Nybo Andersen A-M, et al. Childhood intelligence in relation to adult coronary heart disease and stroke risk: evidence from a Danish birth cohort study. Paediatr Perinat Epidemiol 2005;19:452-9.

2. Batty GD, Wennerstad KM, Smith GD, Modig Wennerstad K, Davey Smith G, et al. Iq in early adulthood and later cancer risk: cohort study of one million Swedish men. Ann Oncol 2007;18:21-8.

3. Mikkelsen SS, Flensborg-Madsen T, Eliasen M, et al. A longitudinal cohort study of intelligence and later hospitalisation with mental disorder. Compr Psychiatry 2014;55:912-9.

4. Wraw C, Deary IJ, Der G, et al. Intelligence in youth and mental health at age 50. Intelligence 2016;58:69-79.

5. Mortensen EL, Sørensen HJ, Jensen $\mathrm{HH}$, et al. lq and mental disorder in young men. Br J Psychiatry 2005;187:407-15.

6. Hatch SL, Jones PB, Kuh D, et al. Childhood cognitive ability and adult mental health in the British 1946 birth cohort. Soc Sci Med 2007;64:2285-96.

7. Batty GD, Deary IJ, Schoon I, et al. Childhood mental ability and adult alcohol intake and alcohol problems: the 1970 British cohort study. Am J Public Health 2008;98:2237-43.

8. Fergusson DM, Horwood LJ, Ridder EM. Show me the child at seven II: childhood intelligence and later outcomes in adolescence and young adulthood. J Child Psychol Psychiatry 2005;46:850-8.

9. Koenen KC, Moffitt TE, Roberts AL, et al. Childhood IQ and adult mental disorders: a test of the cognitive reserve hypothesis. $A m \mathrm{~J}$ Psychiatry 2009;166:50-7.

10. Osler M, Nordentoft M, Andersen A-MN. Childhood social environment and risk of drug and alcohol abuse in a cohort of Danish men born in 1953. Am J Epidemiol 2006;163:654-61.

11. Gale CR, Deary IJ, Boyle SH, et al. Cognitive ability in early adulthood and risk of 5 specific psychiatric disorders in middle age: the Vietnam experience study. Arch Gen Psychiatry 2008;65:1410-8.

12. Batty GD, Deary IJ, Macintyre S. Childhood IQ and life course socioeconomic position in relation to alcohol induced hangovers in adulthood: the Aberdeen children of the 1950s study. J Epidemiol Community Health 2006;60:872-4.

13. David AS, Zammit S, Lewis G, et al. Impairments in cognition across the spectrum of psychiatric disorders: evidence from a Swedish conscript cohort. Schizophr Bull 2008;34:1035-41.

14. Sjölund $S$, Allebeck $P$, Hemmingsson T. Intelligence quotient (IQ) in adolescence and later risk of alcohol-related hospital admissions 
and deaths--37-year follow-up of Swedish conscripts. Addiction 2012;107:89-97.

15. Sjölund S, Hemmingsson T, Gustafsson J-E, et al. lq and alcoholrelated morbidity and mortality among Swedish men and women: the importance of socioeconomic position. J Epidemiol Community Health 2015;69:858-64.

16. Gale CR, Batty GD, Tynelius P, et al. Intelligence in early adulthood and subsequent hospitalization for mental disorders. Epidemiology 2010;21:70-7.

17. Schuckit MA. An overview of genetic influences in alcoholism. $J$ Subst Abuse Treat 2009;36:S5-14.

18. Sørensen HJ, Manzardo AM, Knop J, et al. The contribution of parental alcohol use disorders and other psychiatric illness to the risk of alcohol use disorders in the offspring. Alcohol Clin Exp Res 2011;35:1315-20.

19. Hasin DS, Stinson FS, Ogburn E, et al. Prevalence, correlates, disability, and comorbidity of DSM-IV alcohol abuse and dependence in the United States: results from the National epidemiologic survey on alcohol and related conditions. Arch Gen Psychiatry 2007;64:830-42.

20. Flensborg-Madsen T, Mortensen EL, Knop J, et al. Comorbidity and temporal ordering of alcohol use disorders and other psychiatric disorders: results from a Danish register-based study. Compr Psychiatry 2009;50:307-14.

21. Mortensen EL. The Copenhagen perinatal cohort and the prenatal development project. Int J Risk Saf Med 1997:10:199-202.

22. Zachau-Christiansen B, Ross E. Babies: Human development during the first year. New York, NY: Wiley, 1975.

23. Teasdale TW. The Danish draft board's intelligence test, Børge Priens Prove: psychometric properties and research applications through 50 years. Scand J Psychol 2009;50:633-8.

24. Mortensen EL, Reinisch JM, Teasdale TW. Intelligence as measured by the WAIS and a military draft board group test. Scand J Psychol 1989;30:315-8.

25. Pedersen CB. The Danish civil registration system. Scand J Public Health 2011;39(7 Suppl):22-5.

26. Mors O, Perto GP, Mortensen PB. The Danish psychiatric central research register. Scand J Public Health 2011;39(7 Suppl):54-7.

27. Schmidt M, Schmidt SAJ, Sandegaard JL, et al. The Danish national patient registry: a review of content, data quality, and research potential. Clin Epidemiol 2015;7:449-90.

28. Becker U. Documentation of patient admission in the Alcohol-unit Hvidovre Hospital. WINALCO - a clinical database [in Danish]. Alkoholenheden: Hvidovre Hospital, 2004.
29. Pedersen AB, Mikkelsen EM, Cronin-Fenton D, et al. Missing data and multiple imputation in clinical epidemiological research. Clin Epidemiol 2017;9:157-66.

30. Osler M, Godtfredsen NS, Prescott E. Childhood social circumstances and health behaviour in midlife: the Metropolit 1953 Danish male birth cohort. Int J Epidemiol 2008;37:1367-74.

31. Clouston SAP, Richards M, Cadar D, et al. Educational inequalities in health behaviors at midlife: is there a role for early-life cognition? $J$ Health Soc Behav 2015;56:323-40.

32. Gottfredson LS, Deary IJ. Intelligence predicts health and longevity, but why? Curr Dir Psychol Sci 2004;13:1-4.

33. Wraw C, Der G, Gale CR, et al. Intelligence in youth and health behaviours in middle age. Intelligence 2018;69:71-86.

34. Boden JM, Fergusson DM. Alcohol and depression. Addiction 2011;106:906-14.

35. Chou K-L, Mackenzie CS, Liang K, et al. Three-Year incidence and predictors of first-onset of DSM-IV mood, anxiety, and substance use disorders in older adults: results from wave 2 of the National epidemiologic survey on alcohol and related conditions. J Clin Psychiatry 2011;72:144-55

36. Batty GD, Whitley E, Deary IJ, et al. Psychosis alters association between IQ and future risk of attempted suicide: cohort study of 1,109,475 Swedish men. BMJ 2010;340:c2506.

37. Jester JM, Buu A, Zucker RA. Longitudinal phenotypes for alcoholism: heterogeneity of course, early identifiers, and life course correlates. Dev Psychopathol 2016;28:1531-46.

38. Meier MH, Caspi A, Houts R, et al. Prospective developmental subtypes of alcohol dependence from age 18 to 32 years: implications for nosology, etiology, and intervention. Dev Psychopathol 2013;25:785-800.

39. Grant JD, Heath AC, Bucholz KK, et al. Spousal concordance for alcohol dependence: evidence for assortative mating or spousal interaction effects? Alcoholism Clin Exp Res 2007;31:717-28.

40. Plomin R, Deary IJ. Genetics and intelligence differences: five special findings. Mol Psychiatry 2015;20:98-108.

41. Lawlor DA, Batty GD, Morton SMB, et al. Early life predictors of childhood intelligence: evidence from the Aberdeen children of the 1950s study. J Epidemiol Community Health 2005;59:656-63.

42. Enoch M-A. The role of early life stress as a predictor for alcohol and drug dependence. Psychopharmacology 2011;214:17-31.

43. Enoch M-A. Genetic influences on the development of alcoholism. Curr Psychiatry Rep 2013:15:412.

44. Deak JD, Miller AP, Gizer IR. Genetics of alcohol use disorder: a review. Curr Opin Psychol 2019;27:56-61. 